\title{
Dimensão fractal de séries de variáveis micrometeorológicas em uma floresta inundável no Pantanal de mato grosso
}

\author{
Fractal dimension of micrometeorological variables series in a floodplain forest in the Pantanal \\ of Mato Grosso
}

\author{
Geison Jader Mello ${ }^{1}$, Marcelo Sacardi Biudes², Raphael Souza Rosa Gomes ${ }^{3}$, \\ Nadja Gomes Machado 2,4, Osvaldo Alves Pereira e José de Souza Nogueira \\ ${ }^{1}$ Doutor, Instituto Federal de Mato Grosso, Jaciara, Brasil - \\ geison.mello@svc.ifmt.edu.br \\ ${ }^{2}$ Doutor, Instituto de Física, Universidade Federal de Mato Grosso, Cuiabá, Brasil \\ marcelo@fisica.ufmt.br; nogueira@ufmt.br \\ ${ }^{3}$ Doutor, Instituto de Computação, Universidade Federal de Mato Grosso, Cuiabá, Brasil -thesivis@gmail.com \\ ${ }^{4}$ Doutora, Laboratório de Biologia da Conservação, Instituto Federal de Mato Grosso, Cuiabá, Brasil \\ nadja.machado@blv.ifmt.edu.br \\ ${ }^{5}$ Doutor, Programa de Pós-Graduação em Ciências Ambientais, Universidade de Cuiabá, Cuiabá, Brasil \\ professor-fu@hotmail.com
}

\begin{abstract}
Resumo
O estudo da dinâmica das variáveis micrometeorológicas é importante para a compreensão do funcionamento dos ecossistemas. A evolução desses sistemas é de difícil compreensão quando analisados a partir de métodos tradicionais lineares. Isto implica a necessidade de métodos de observação e de descrição teórica dos fenômenos que leve em conta a não linearidade nas equações de movimento que regem a dinâmica de tais sistemas complexos. $O$ objetivo desse trabalho foi determinar a dimensão fractal de séries de variáveis micrometeorológicas em uma floresta inundável no Pantanal Mato-grossense. As estimativas da dimensão fractal Dc e dimensão de imersão $m$ das variáveis micrometeorológica da radiação solar global, radiação fotossinteticamente ativa, temperatura e umidade relativa do ar, evapotranspiração, fluxo de $\mathrm{CO} 2$ e concentração de $\mathrm{CO} 2$ evidenciam a existência de acoplamento de subsistemas não lineares de baixa dimensão governados por atratores estranhos, dado o seu número e a variedade das interações.
\end{abstract}

Palavras-chave: Sistema não linear. Sistema dinâmico. Balanço de energia. Reconstrução do espaço de fase.

\begin{abstract}
The study of the micrometeorological variables dynamics is important for understanding the ecosystems function. The evolution of these systems is difficult to comprehend when they have been analyzed by traditional linear methods. This implies the need for the use of methods of observation and theoretical description of the phenomena that takes into account the non-linearity of the equations of motion of the dynamics of such complex systems. The objective of this study was to determine the fractal dimension of micrometeorological variables series in a floodedplain forest in the Pantanal of Mato Grosso. Estimates of the fractal dimension Dc and dimension of immersion $m$ of micrometeorological variables of global radiation, photosynthetically active radiation, temperature and relative humidity, evapotranspiration, $\mathrm{CO} 2$ flow and $\mathrm{CO} 2$ concentration showed the existence of coupling nonlinear subsystems low dimension ruled by strange attractors, because the number and the variety of interactions.
\end{abstract}

Keywords: Nonlinear system. Dynamic system. Energy balance. Reconstruction of phase space. 


\section{Introdução}

Adinâmica dos ecossistemas tropicais tem recebido considerável importância pela necessidade de compreender os efeitos das mudanças da cobertura terrestre sobre os ciclos biogeoquímicos regionais e o papel de ecossistemas terrestres tropicais nas trocas de massa e energia (BIUDES et al., 2015).

Áreas alagáveis tropicais ocupam 5 a 7\% da superfície terrestre (NEUE et al., 1997). O Pantanal, que é considerado elo entre o Cerrado, a Amazônia e os Chacos Boliviano e Paraguaio (JUNK e NUNES DA CUNHA, 2005) apresenta o pulso de inundação como principal força moderadora da paisagem devido à baixa drenagem dos solos (JUNK, 2002). As oscilações nos níveis de inundação são causadas pela variação pluviométrica e mudanças na dinâmica sedimentológica ao longo do tempo (COLLISCHONN et al., 2001), o que funciona como filtro ao estabelecimento e desenvolvimento de algumas espécies de plantas (ARIEIRA e NUNES DA CUNHA, 2006). Esta condição tem levado à permanência de elevada quantidade de espécies pioneiras no Pantanal, que mantêm suas populações em altas densidades, de acordo com os requerimentos fisiológicos e ecológicos da espécie (JUNK et al., 2006).

Algumas espécies podem servir como indicador de mudanças ambientais por meio de diferentes respostas às condições de habitat. O cambará (Vochysia divergens Pohl), por exemplo, se destaca por apresentar características ecológicas e fisiológicas que favorecem seu rápido crescimento e dominância em solo nu e em pastagens sazonalmente inundadas, o que indica capacidade de colonizar áreas perturbadas. Entre suas principais características que influenciam na formação de estandes monodominantes, destaca-se a elevada taxa de crescimento sob intensa luminosidade, tolerância à condição de prolongado alagamento, capacidade de suas plântulas em manter folhas intactas debaixo da superfície da água e elevada produção de sementes dispersadas pelo vento (NUNES DA CUNHA e JUNK, 2004).

A comunidade monodominante de cambará, localmente chamada de cambarazal, apresenta peculiar dinâ- mica das variáveis micrometeorológicas. Por exemplo, elevada evapotranspiração durante o ano todo, por ter elevada disponibilidade hídrica durante a estação chuvosa e por ser capaz de acessar o lençol freático superficial com maior eficiência durante o período de estiagem (BIUDES et al., 2009; 2015). O seu rápido espalhamento sobre campos sazonalmente inundados pode alterar a estrutura química e física do solo, indicando que sua invasão fundamentalmente altera o estoque de carbono e nutrientes do solo pantaneiro (VOURLITIS et al., 2011).

A complexidade dos ecossistemas e sua variedade de subsistemas em interação motivam a análise não linear das séries temporais provenientes de florestas (MELLO et al., 2013). Pesquisas recentes têm abordado a natureza caótica dos dados coletados em florestas tropicais contribuindo para o entendimento e modelagem da interação biosfera-atmosfera (CAMPANHARO et al., 2008). Do ponto de vista dos sistemas dinâmicos (PEITGEN et al., 2004), várias pesquisas com séries temporais climáticas identificaram a possível existência de atratores climáticos de baixa dimensão caótica e indicam o número mínimo de variáveis presentes na evolução do sistema (GALLEGO et al., 2001; XIN et al., 2001). Esta abordagem contribui com um passo em direção a um conhecimento adicional seja da natureza intrínseca do ambiente onde os dados são coletados seja dos fenômenos ora observados.

Sendo assim, o objetivo desse trabalho foi determinar a dimensão fractal de séries de variáveis micrometeorológicas em uma floresta inundável no Pantanal Mato-grossense.

Reconstrução do espaço de fase das variáveis

As séries temporais, como exemplo da Figura 1A, são entendidas como uma sequência de observações ao longo do tempo, e podem fornecer informações valiosas sobre a dinâmica subjacente da qual são provenientes.

Admitindo-se que uma série temporal é obtida de um sistema dinâmico com um número $m$ de variáveis desconhecidas, é possível extrair mais informações dela do que apenas o padrão de comportamento da série em si. Pois nela contém impressões de outras variáveis ocultas do sistema e que são acopladas ao seu espaço de fase $m$ dimensional (Figura 1B).
A.

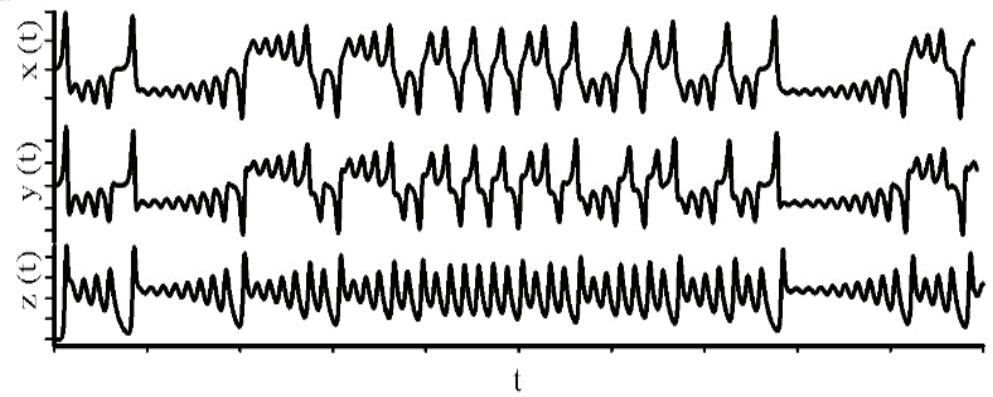

B.

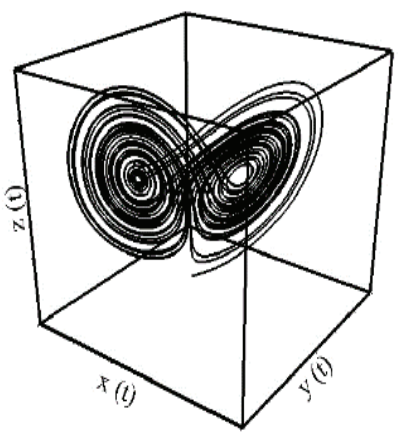

Figura 1 - (A) solução numérica do sistema de equações de Lorenz (36) para x(t), y(t) e $z(t)$ e (B) representação do espaço de fase tridimensional do atrator estranho de Lorenz (parâmetros de controle $\rho=25, \sigma=10, \beta=8 / 3$ ) 


\section{Método das defasagens temporais}

Umas das mais importantes técnicas de reconstrução do espaço de fase para identificar o atrator a partir de uma única série temporal é o método das defasagens temporais proposto por Takens (1981). O espaço reconstruído apresenta uma suave variação de coordenada em relação ao espaço original, preservando os invariantes geométricos do sistema, como a dimensão fractal do atrator por exemplo (Figura 2C). Este poderoso método utiliza apenas uma série temporal do sistema dinâmico, $\mathrm{X}_{0}(\mathrm{t})$, na qual o atrator está oculto para obter (ou reconstruir) as outras variáveis $\left\{X_{k}(t)\right\}$ (sendo $k=1, \ldots, n-1$ ) conhecendo somente $X_{0}(t)$. Então, a série temporal original $X_{0}(t)$ é desdobrada em deslocamentos temporais sucessivos de defasagem fixa $\tau(\tau=m \Delta \mathrm{t}$, onde $m$ é um número inteiro e $\Delta$ t é um intervalo entre sucessivas amostras) para " $n$ " pontos equidistantes do conjunto de dados, isto é,

$$
\begin{aligned}
& X_{0}: X_{0\left(t_{1}\right)}, \ldots, X_{0\left(t_{n}\right)} \\
& X_{1}: X_{0\left(t_{1}+\tau\right)}, \ldots, X_{0\left(t_{n}+\tau\right)} \\
& X_{n-1}: X_{0\left(t_{1}+(n-1) \tau\right)}, \ldots, X_{0\left(t_{n}+(n-1) \tau\right)}
\end{aligned}
$$

A melhor escolha de $\tau$ deve apresentar uma independência linear entre a série original e as séries defasadas, ou seja, a menor autocorrelação, no intuito de obter a maior informação possível do sistema estudado. A reconstrução do atrator com coordenadas de atraso temporal não é um processo automático, logo é importante a escolha de um $\tau$ apropriado (Figura 3) com afirma Baker e Gollub (1996).

\section{Determinação do $\tau$ pela função autocorrelação}

Em geral, os dados vizinhos em uma série temporal são correlacionados, e para extrair o máximo de informação desta série utiliza-se a função autocorrelação (acf $(\tau))$, que estima o grau de correlação de uma variável em um dado instante consigo mesma à medida que o tempo passa, ou seja, a dependência entre o mesmo sinal defasado. $\mathrm{O}$ tempo de defasagem, $\tau$ adequado, é encontrado quando a função $a c f(\tau)$ atinge o valor zero ou o primeiro mínimo, característica da interdependência entre e (Equação 4).

$$
\operatorname{acf}(\tau)=\frac{1}{n-\tau} \sum_{t=1}^{n-\tau} \frac{\left(X_{(t)}-\bar{X}\right)\left(X_{(t+\tau)}-\bar{X}\right)}{\sigma^{2}}
$$

em que $n$ é o número de dados da série, $\bar{X}$ sua média e $\sigma^{2}$ sua variância. Plota-se $a c f(\tau)$ versus $\tau$ para visualizar a forma como a dependência da série cai com o $\tau$ entre pontos da série. Para séries periódicas e quasiperiódicas a $a c f(\tau)$ permanece diferente de zero e igualmente periódica quando o tempo tende ao infinito, pois o sinal volta a parecer consigo após um período (Figura 3A). Para sistemas altamente não lineares $\operatorname{acf}(\tau)$ tende a zero quando $\tau$ tende ao infinito (Figura 3B).

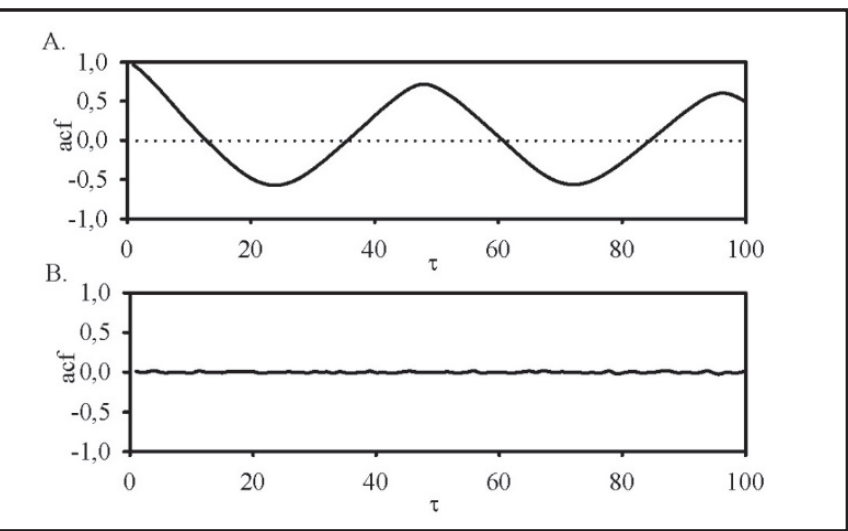

Figura 3 - (A) autocorrelação da série de $\mathrm{CO}_{2}(35 \mathrm{~m})$ e (B) de uma série de dados aleatórios

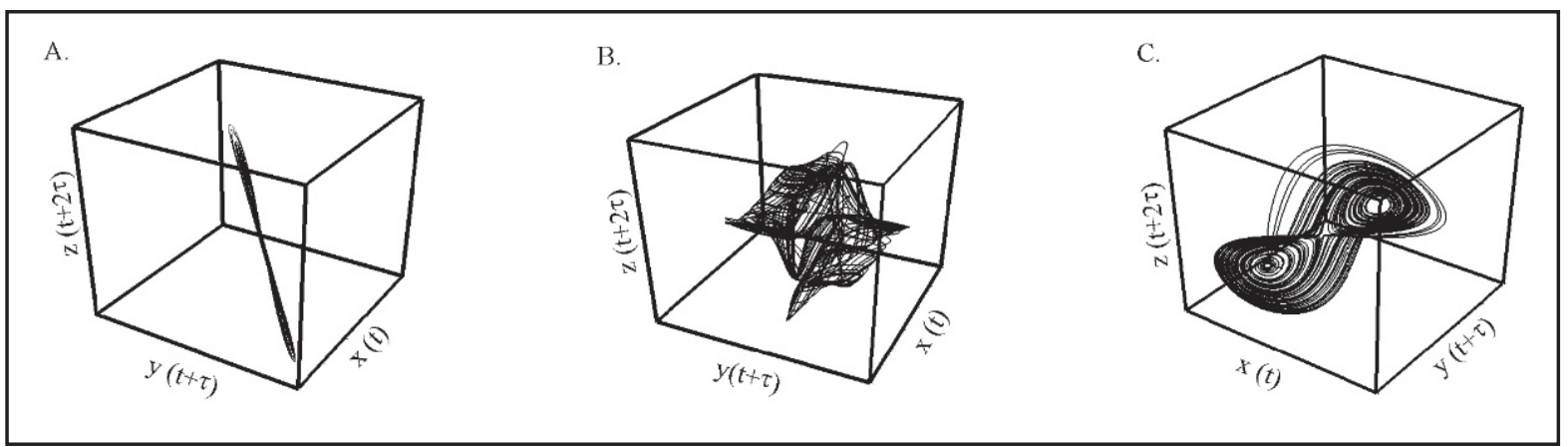

Figura 2 - Efeito da defasagem temporal $\tau$ na reconstrução do atrator de Lorenz (1963) para (A) uma defasagem muito pequena $(\tau=2)$, (B) uma defasagem muito grande $(\tau=50)$ e para $(C)$ uma defasagem adequada $(\tau=10)$ 


\section{Atratores estranhos e dimensão fractal}

A dimensão fractal $(D c)$ é uma medida comumente usada para medir a "estranheza" dos atratores, assim como o número de graus de liberdade e informações estatísticas sobre o sistema. Neste trabalho foi aplicado o algoritmo de Grassberger e Procaccia (1983) que é o mais amplamente utilizado, dentre os diferentes procedimentos desenvolvidos para computar a dimensão fractal. A $D c$ também provê o número de variáveis independentes necessárias à descrição da evolução temporal da dinâmica do sistema com a dimensão de imersão $M(37)$ que tem como limite superior $2 D c+1$ para modelar um sistema dinâmico.

Em um espaço de fase m-dimensional, a função correlação integrante $C(r)$ do atrator é dada pela Equação (5) (GRASSBERGER e PROCACCIA, 1983).

$$
C(r)=\frac{1}{n^{2}} \sum_{\substack{i, j=1 \\ i \neq j}}^{n} \theta\left(r-\left|\mathrm{X}_{\mathrm{i}}-\mathrm{X}_{j}\right|\right)
$$

em que $\theta$ é a função Heaviside, $\theta(x)=0$ se $x<0$, e $\theta(x)$ $=1$ se $x>0$.

A partir de uma pequena correlação $\varepsilon$, sonda-se a estrutura do atrator. Se esta é uma linha, o número de pontos dentro de uma sondagem à distância $r$ de um ponto deve ser proporcional ao $r / \varepsilon$. Se for uma superfície, este número deve ser proporcional a $(r / \varepsilon)^{2} \mathrm{e}$, de forma geral, se for uma dimensão $d$ deve ser proporcional a $(r / \varepsilon)^{d}$. Logo, para $r$ relativamente pequeno, deverá variar conforme a Equação (6).

$$
C(r) \sim r^{d}
$$

Assim, como a dimensão de correlação $D c$ do atrator é aproximadamente igual à dimensão fractal $d$, ela é dada pela declinação da relação entre $\ln C(r)$ e $\ln r$ para um valor de $r$ infinitesimal crescente até a integração total do atrator. Ou seja, a dimensão fractal é obtida do prolongamento linear da figura "joelho" (Figura 4A e 4B) do qual são extraídos os coeficientes lineares (Figura 4C; Equação 7).

$$
\ln C(r)=d \ln r
$$

Afere-se a dimensionalidade mínima, $m$, do espaço de fase dentro do qual o atrator mencionado está embutido. $\mathrm{O}$ parâmetro $m$ define o número mínimo de variáveis que devem ser consideradas na descrição da dinâmica do sistema, ou seja, o número mínimo de equações diferenciais de primeira ordem que podem conter as características qualitativas do sistema dinâmico estudado (GRASSBERGER e PROCACCIA, 1983). Importante ressaltar que $D_{c}$ é necessariamente menor que $m$.

\section{Material e Métodos}

\section{Localização da área experimental}

A área experimental localiza-se na Reserva Particular do Patrimônio Natural - RPPN SESC - Pantanal, município de Barão de Melgaço - MT (Figura 5), distante 160 km de Cuiabá, Mato Grosso, na região Centro-Oeste do Brasil, onde está instalada uma torre micrometeorológica

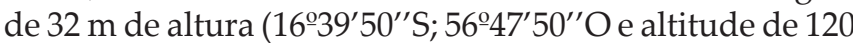
$\mathrm{m})$. Esta área apresenta uma floresta monodominante de Cambará (Vochysia divergens Pohl), conhecida localmente como cambarazal, com altura do dossel variando entre 28 a 30 m (BIUDES et al., 2012). O solo foi classificado como GLEISSOLO HÁPLICO Ta Distrófico.

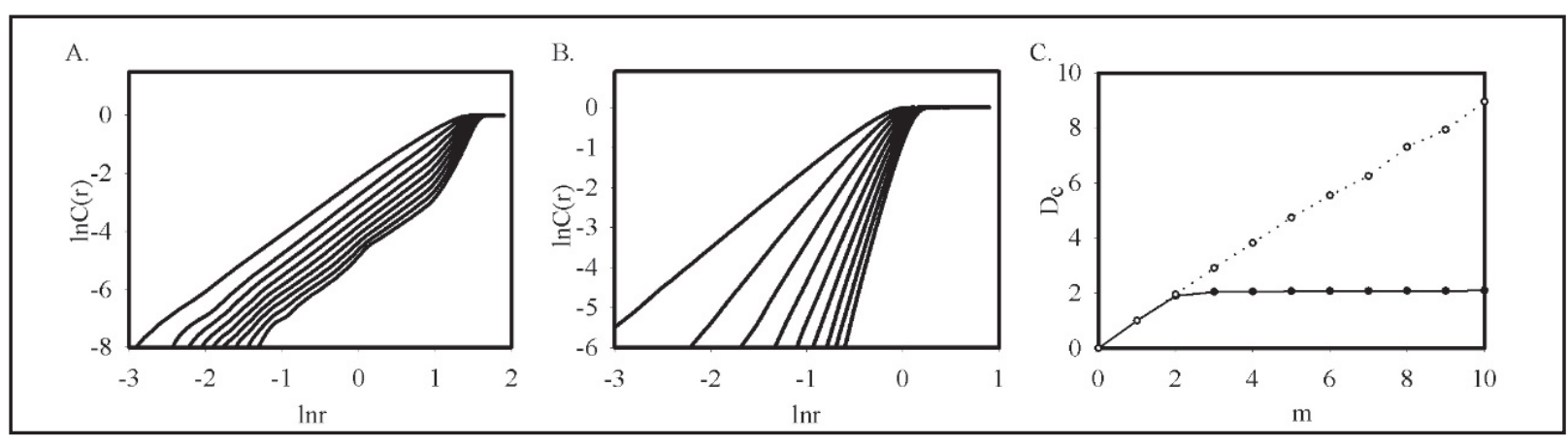

Figura 4: (a) vs. dos valores crescentes de $m$ do atrator reconstruído a partir da série $\mathrm{x}$ de Lorenz e (b) vs. da uma série de dados aleatórios; e (c) saturação da dimensão de correlação versus dimensão de imersão m do atrator reconstruído a partir da série $x$ de Lorenz $\operatorname{com} D_{c} \approx 2,05 \pm 0,01 e m=3(\bullet)$ e a instauração para a série aleatória com dimensão infinita (o). 


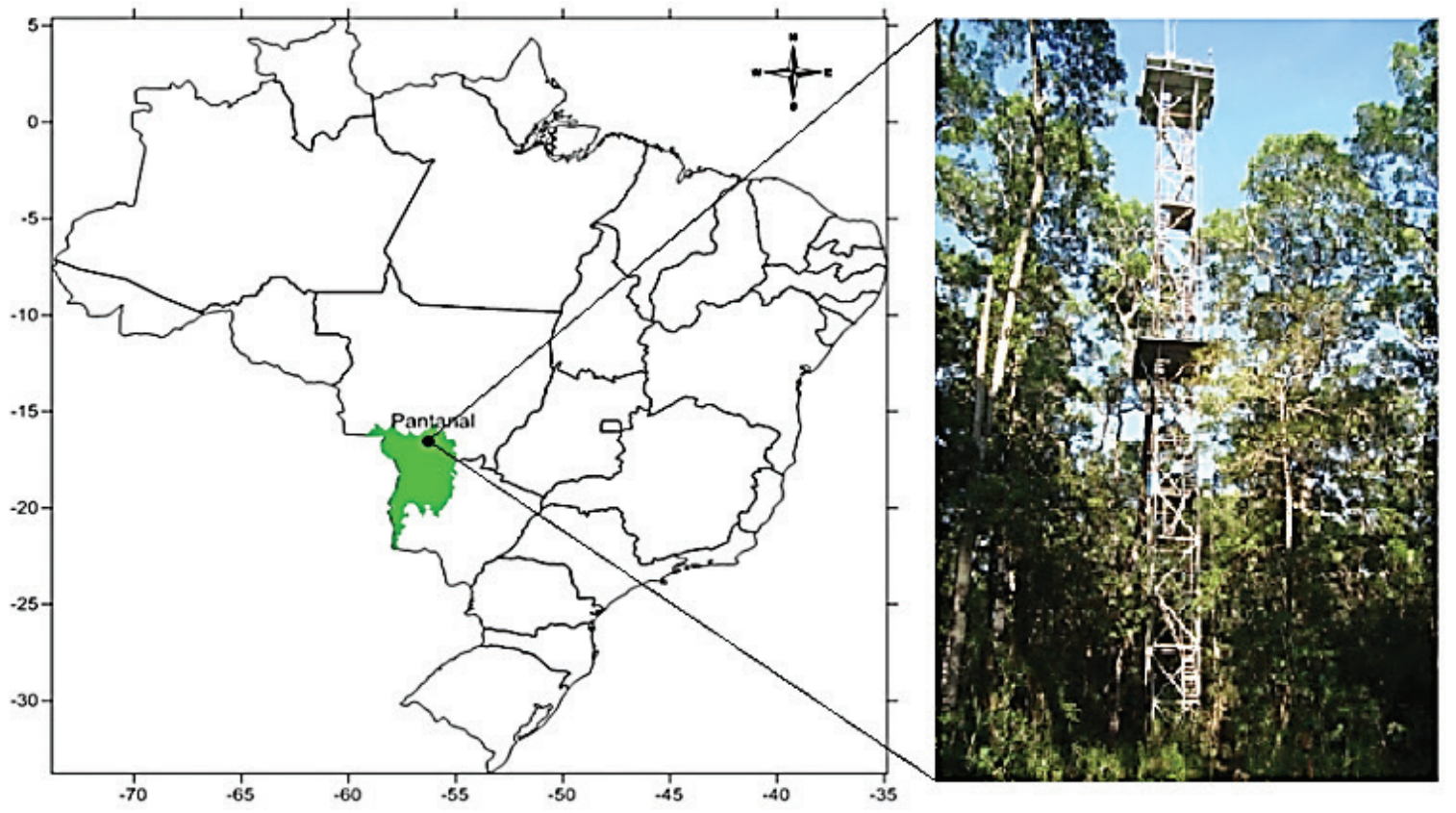

Figura 5 - Indicação da área de vegetação monodominante de Cambará no Pantanal - RPPN SESC, no município de Barão de Melgaço-MT, Brasil, e a torre micrometeorológica

O clima é Aw segundo a classificação de Köppen, com média anual de temperatura do ar é de $25,6{ }^{\circ} \mathrm{C}$ e de umidade relativa do ar é de $73,1 \%$, precipitação média anual é de $1342 \mathrm{~mm}$, com precipitação inferior a $15 \mathrm{~mm}$ no período seco de junho a início de setembro e estação chuvosa com $86 \%$ da precipitação acumulada anual (10; 26). Frequentemente a inundação ocorre entre dezembro e março (0,6 - 1,5m de altura), que acompanha a estação chuvosa e a oscilação anual do nível d'água do rio Cuiabá, sendo altamente influenciada pela precipitação local (NUNES DA CUNHA e JUNK, 2004).

O período de análise dos dados neste trabalho correspondem medidas realizadas entre 21 de dezembro 2008 a 20 de janeiro de 2009.

\section{Instrumentos micrometeorológicos utilizados}

Os instrumentos utilizados para coleta de dados foram um analisador de gás de circuito fechado para medir a concentração de $\mathrm{CO}_{2}$ (LI-820, LI-COR, Inc.) nas alturas 3; 12; 20; 29; 33; 35 m; um saldo radiômetro (Net Radiometer, Kipp \& Zonen Delft, Inc., Holland) para medir o saldo de radiação a $33 \mathrm{~m}$ de altura; um piranômetro (LI-200SA, LI-COR, Inc., Nebraska, USA) para medir a radiação solar global a $33 \mathrm{~m}$ de altura; um sensor quântico (LI-190, LICOR, Inc., Nebraska, USA) para medir a radiação fotossinteticamente ativa incidente (PARi) a $33 \mathrm{~m}$ de altura. A temperatura e a umidade relativa do ar foram medidas por meio de dois termohigrômetros (HMP 45 C, Vaisala, Inc., Helsinki, Finland) instalados a 33,7 e $37,7 \mathrm{~m}$, e o fluxo de calor no solo por meio de dois fluxímetros (HFT-3.1, REBS, Inc., Seattle, Washington) instalados a $2 \mathrm{~cm}$ de profundidade.
Os dados foram processados e armazenados por data loggers (CR 10X, Campbell Scientific, Inc., Ogden, Utah) com média de 30 minutos. Para aumentar o número de canais de entrada no registrador foi utilizada uma placa multiplexadora (AM16/32A-ST-SW, Campbell Scientific, Inc., Ogden, Utah).

\section{Estimativa da evapotranspiração e da troca líquida de $\mathrm{CO}_{2}$}

A evapotranspiração (Equação 8) foi calculada pelo método da razão de Bowen (BIUDES et al., 2009; 2015) a partir do cálculo do fluxo de calor latente (LE) (Equação 9) e do calor latente de vaporização $(\lambda)$ (Equação 10).

$$
\begin{aligned}
& E T=\frac{L E}{\lambda} \\
& L E=\frac{R n-G-\Delta S}{1+\beta} \\
& \lambda=1.919 \times 10^{6}\left(\frac{T+273.16}{(T+273.16)-33.91}\right)^{2}
\end{aligned}
$$

em que Rn é o saldo de radiação $\left(\mathrm{W} \mathrm{m}^{-2}\right)$ medido sobre o dossel do cambarazal, G é o fluxo de calor no solo (W $\left.\mathrm{m}^{-2}\right), \Delta \mathrm{s}$ é o calor estocado no ar e biomassa do dossel $\left(\mathrm{W} \mathrm{m}^{-2}\right)$ estimado pela parametrização de Moore e Fisch (1986) e $\beta$ é a razão de Bowen estimada pela Equação (11) e $T$ é a temperatura do ar $\left({ }^{\circ} \mathrm{C}\right)$.

$$
\beta=\left(\frac{C_{p}}{\lambda 0.622}\right)\left(\frac{\Delta T}{\Delta e}\right)
$$


em que $\beta$ é o calor específico do ar à pressão constante $\left(1.00467 \mathrm{~J} \mathrm{~g}^{-1} \mathrm{~K}^{-1}\right), 0,622$ é a razão do peso molecular da água e do ar, e é a diferença de temperatura $\left({ }^{\circ} \mathrm{C}\right)$ e de pressão de vapor d'água $(\mathrm{kPa})$ entre os dois níveis.

O filtro proposto por Perez et al. (1999) e revisado por $\mathrm{Hu}$ et al. (2014) foi aplicado sobre as estimativas do LE. O método da razão de Bowen falha quando (1) a resolução instrumental é inadequada para calcular os gradientes de temperatura e pressão de vapor d'água (UNLAND et al., 1996), (2) quando a atmosfera é neutra, durante o amanhecer e o anoitecer, o que causa $\beta \approx-1$ (ORTEGA-FARIAS et al., 1996) e a evapotranspiração tende ao infinito, e (3) em mudança abrupta dos gradientes ou da disponibilidade de energia para o ambiente (PEREZ et al., 1999). As estimativas do LE que não se enquadraram no critério proposto por Perez et al. (1999) foram retirados. Para preenchimento das falhas após o critério de Perez et al. (1999) foi usado uma regressão linear entre os valores de LE e as medidas de Rn-G (GRACE et al., 1996).

O coeficiente de difusão turbulenta do calor sensível $\left(; \mathrm{m}^{2} \mathrm{~s}^{-1}\right)$, foi calculado pela Equação (12).

$$
K_{H}=\left(\frac{H}{\rho C_{p}}\right) \cdot\left(\frac{\Delta z}{\Delta T}\right)
$$

em que é o fluxo de calor sensível $\left(\mathrm{J} \mathrm{m}^{-2} \mathrm{~s}^{-1}\right)$, é a densidade do ar $\left(\mathrm{mg} \mathrm{m}^{-3}\right), \mathrm{C}_{\mathrm{p}}$ é o calor específico à pressão constante $\left(1,00467 \mathrm{~J} \mathrm{~g}^{-1} \mathrm{k}^{-1}\right)$ e $\Delta \mathrm{z}$ e $\Delta \mathrm{T}$ são a diferença de altura $(\mathrm{m})$ e a diferença de temperatura do ar $\left({ }^{\circ} \mathrm{C}\right)$ entre os termohigrômetros superior e inferior.

A troca líquida de $\mathrm{CO}_{2}$ do ecossistema consiste na diferença entre o $\mathrm{CO}_{2}$ absorvido na fotossíntese e a liberação durante a respiração pela vegetação e pelos microrganismos do solo (ARAÚJO et al., 2010). A troca líquida de $\mathrm{CO}_{2}$ do cambarazal $\left(\mathrm{A}_{\mathrm{cf}} ; \mu \mathrm{mol} \mathrm{m} \mathrm{m}^{-2} \mathrm{~s}^{-1}\right)$ foi estimada pela Equação (13).

$$
A_{c f}=K_{c} \cdot\left(\frac{\Delta C}{\Delta z}\right) \cdot\left(\frac{1000}{44}\right)
$$

em que é o coeficiente de difusão turbulenta do fluxo de $\mathrm{CO}_{2}\left(\mathrm{~m}^{2} \mathrm{~s}^{-1}\right), \Delta \mathrm{C}$ é a diferença de concentração de $\mathrm{CO}_{2}$ $\left(\mathrm{mg} \mathrm{m}^{-3}\right)$ entre as duas alturas consideradas e (1000/44) é um fator que converte a unidade do fluxo de $\mathrm{CO}_{2}$ de $\mathrm{mg}$ $\mathrm{m}^{-2} \mathrm{~s}^{-1} \mathrm{em} \mu \mathrm{mol} \mathrm{m} \mathrm{m}^{-2} \mathrm{~s}^{-1}$ (WOLF, et al., 2008). Aplicando-se a teoria da similaridade para o transporte turbulento, .

A concentração de $\mathrm{CO}_{2}$ em cada altura $\left(\mathrm{C}_{\mathrm{ppm}} ; \mathrm{ppm}\right)$ foi corrigida pelo fator de temperatura $\left(T ;{ }^{\circ} \mathrm{C}\right)$ e pressão atmosférica $\left(\mathrm{P}_{\mathrm{atm}} ;\right.$ mbar $)$ pela Equação (14).

$$
C=C_{p p m} 1,964\left(\frac{273,15}{T+273,15}\right)\left(\frac{P_{a t m}}{1013}\right)
$$

\section{Calculo da função autocorrelação $(\operatorname{acf} f(\tau))$, tempo de defasagem $(\tau)$, dimensão fractal $(D c)$ e da dimensão de imersão $(m)$}

A função autocorrelação $(a c f(\tau))$, tempo de defasagem $(\tau)$, dimensão fractal $(D c)$ e da dimensão de imersão $(m)$ foram calculados no Matlab®, utilizando um pacote definido como TOCSY - Toolbox for Complex Systems pelo Potsdam Institute for Climate Impact Research (PIK) (disponível em <http://tocsy.agnld.uni-potsdam. de> acesso em 27/04/2011).

\section{Resultados e Discussão}

As séries temporais das variáveis foram submetidas à função autocorrelação $(a c f(\tau))$ para determinar o tempo de defasagem $\tau$ adequado para a reconstrução dos atratores. A $\operatorname{acf}(\tau)$ variou de 5 a 10,5 horas, ou seja, apresentou uma defasagem temporal $\tau$ entre 10 e 21 (Tabela 1). Estes parâmetros possibilitam inferir quanto um dado da série, em determinado instante, está correlacionado com o seu futuro. Também permite avaliar o quão rápido é a mudança no padrão de comportamento das variáveis. A variável que primeiro chegou a zero na $\operatorname{acf}(\tau)$ foi a evapotranspiração, seguida pela radiação solar global e da radiação fotossinteticamente ativa que são fortemente correlacionadas. A umidade relativa do ar foi a que levou mais tempo para atingir o primeiro zero na autocorrelação, muito possivelmente pela sua dependência (ou retroalimentação) vinculada à evapotranspiração.

Tabela 1: Resultados da função autocorrelação $(\operatorname{acf}(\tau))$, tempo de defasagem $(\tau)$, dimensão fractal

$(D c)$ e da dimensão de imersão $(m)$ das séries temporais de temperatura do ar (T), umidade relativa do ar (Rh), radiação solar global (RG), radiação fotossinteticamente ativa (PARi), evapotranspiração (Et), fluxo de $\mathrm{CO}_{2}\left(\mathrm{CO}_{2}\right.$ flux $)$ e concentração de $\mathrm{CO}_{2}$ em deferentes alturas.

\begin{tabular}{ccccc}
\hline Variáveis & $\boldsymbol{a c} \boldsymbol{f}(\boldsymbol{\tau})$ & $\boldsymbol{\tau}$ & $\boldsymbol{D} \boldsymbol{c}$ & $\boldsymbol{m}$ \\
\hline $\mathrm{T}$ & $8 \mathrm{~h}$ & 16 & $3,05 \pm 0,13$ & 6 \\
$\mathrm{Rh}$ & $10,5 \mathrm{~h}$ & 21 & $3,00 \pm 0,01$ & 6 \\
$\mathrm{RG}$ & $5,5 \mathrm{~h}$ & 11 & $2,52 \pm 0,01$ & 9 \\
PARi & $5,5 \mathrm{~h}$ & 11 & $3,86 \pm 0,05$ & 17 \\
$\mathrm{Et}$ & $5 \mathrm{~h}$ & 10 & $4,38 \pm 0,07$ & 19 \\
$\mathrm{CO}_{2}$ flux & $7 \mathrm{~h}$ & 14 & $5,94 \pm 0,09$ & 8 \\
$\begin{array}{c}\text { Concentração } \\
\text { de } \mathrm{CO}_{2}(*)\end{array}$ & $6,5 \mathrm{~h}$ & 13 & $3,50 \pm 0,09$ & 6 \\
\hline
\end{tabular}

*mesmo valor para as alturas de 35, 33, 32,29, 20,12 e 3 m. 

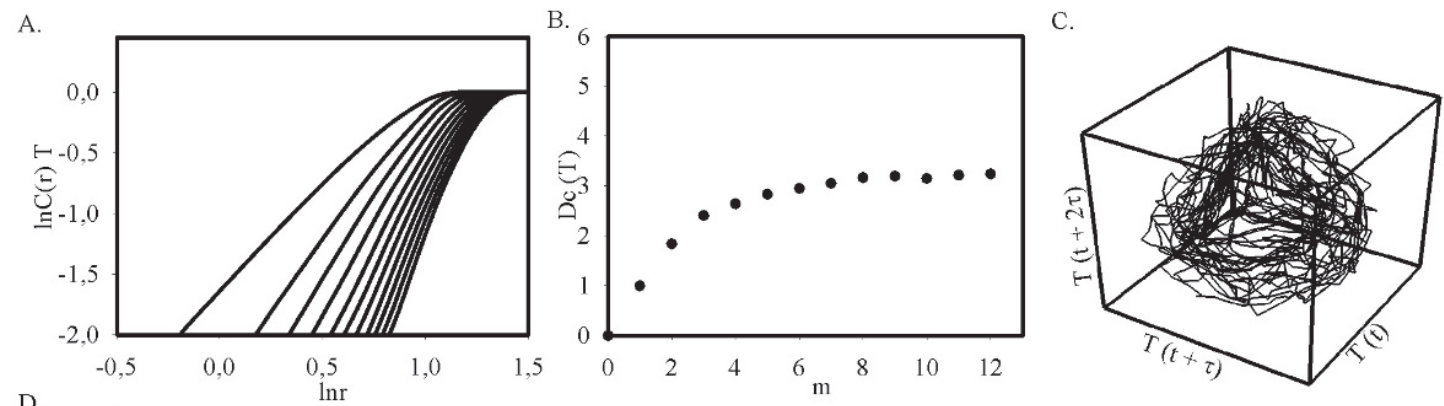

D.
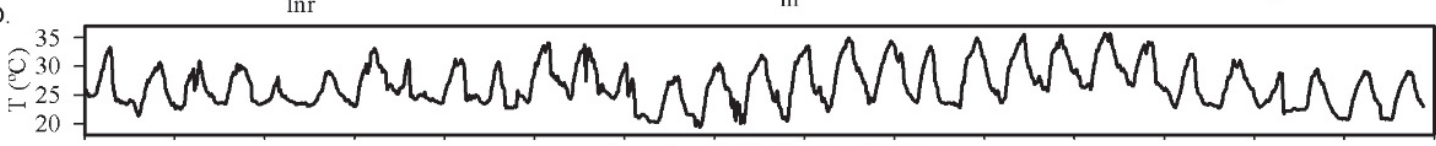

dezembro 2008 / janeiro 2009
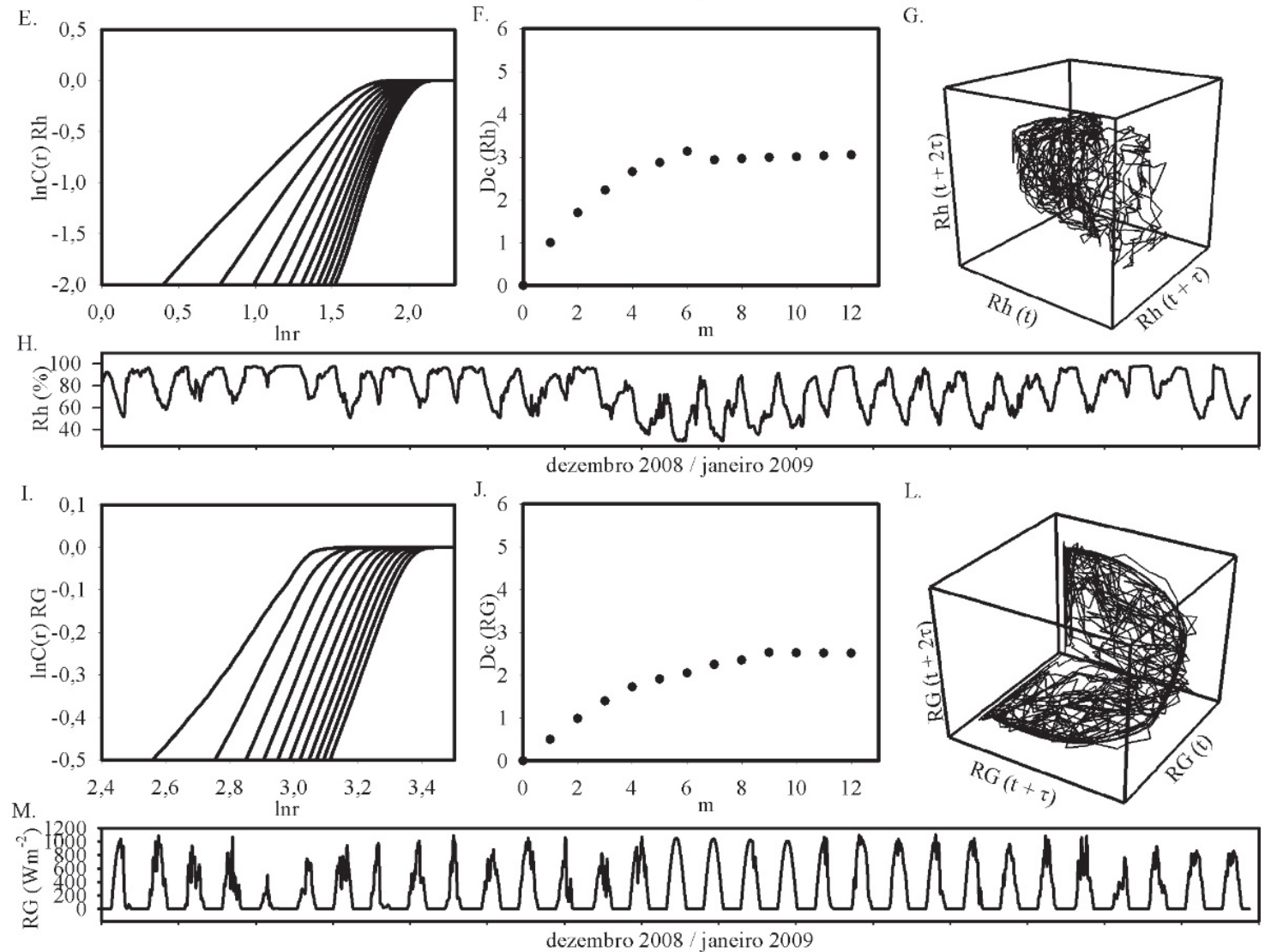

Figura 6: Respectivamente (A), (E) e (I) ) vs. , (B), (F) e (J) versus , (C), (G) e (L) imersão tridimensional do atrator da série temporal da (D) temperatura do ar (T), (H) umidade relativa do ar (Rh) e (M) radiação solar global (RG).

Depois dos cálculos das defasagens temporais de cada uma das séries temporais, o atrator associado a essa variável foi reconstruído, calculando a dimensão fractal $(D c)$ e a dimensão de imersão $(m)$. Para a dimensão de imersão $m$ foram usados valores padrões de 2 a $12 \mathrm{em}$ quase todas as séries, com exceção da radiação fotossinteticamente ativa e da evapotransiração que necessitaram de 17 e 19 dimensões de imersão, respectivamente, para que se pudesse detectar a saturação de $D c$ versus $m$.

Nas Figuras 6A, 6E, 6I, 7A, 7E e 7I observam-se as correlações integrais das séries temporais das variáveis micrometeorológicas versus o raio $\mathrm{r}$ da hiperesfera de sondagem, em diferentes dimensões de imersão m. Estas curvas possuem seções lineares cujos valores das inclinações (os coeficientes angulares dos seguimentos lineares das curvas), calculados por regressão linear simples, saturam ou não quando as dimensões de imersão são incrementadas. Quando saturados os valores de versus, resultam nas dimensões fractais dos atratores, que podem ser observados nas Figuras 6B, 6F, 6J, 7B, 7F, 7J e na Figura 8 para a concentração de $\mathrm{CO}_{2}$ em diferentes alturas.

Teoricamente, o algoritmo de Grassberge e Procaccia (1983) provê uma medida do número de variáveis inde- 


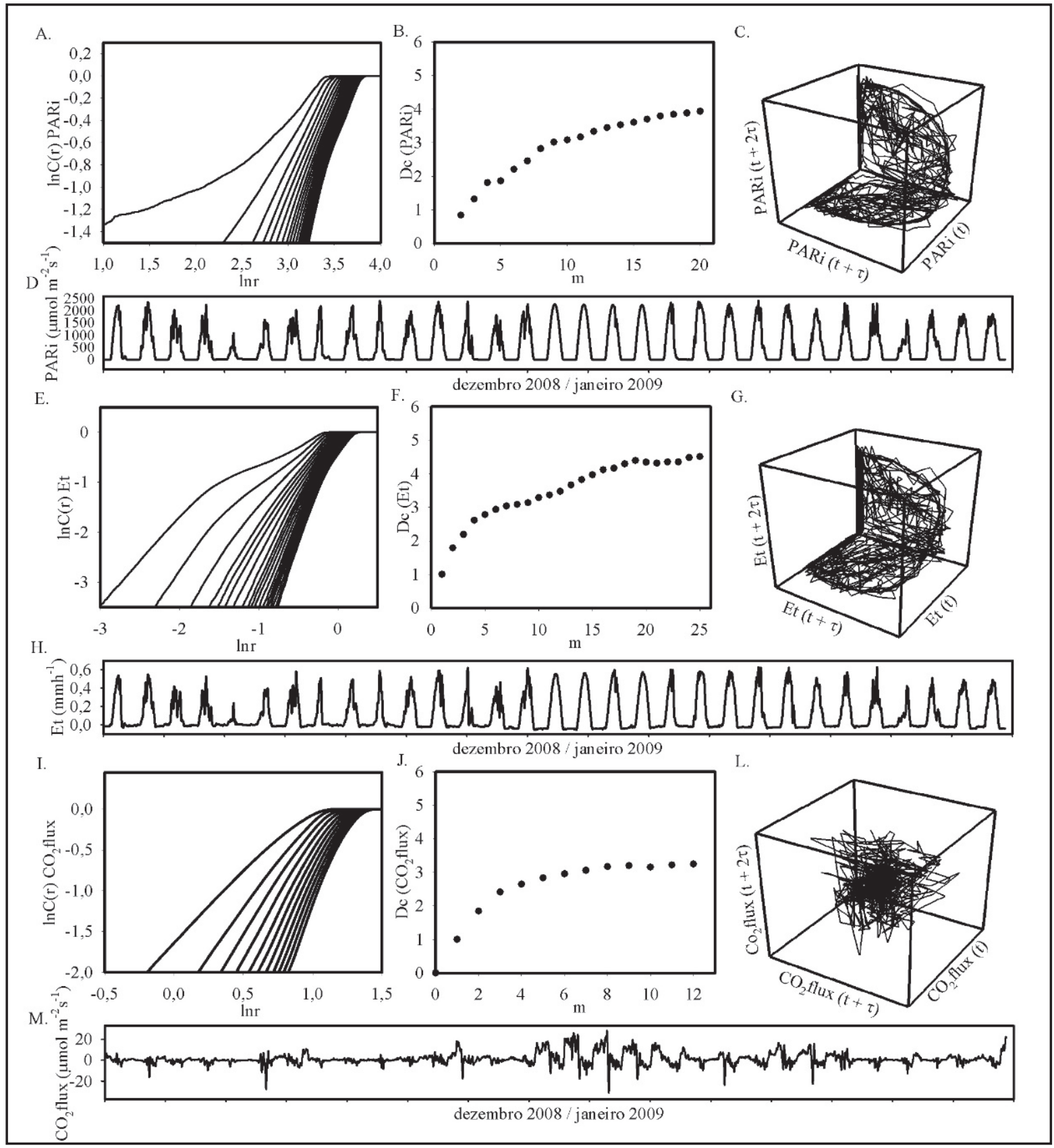

Figura 7: Respectivamente (A), (E) e (I) ) vs. , (B), (F) e (J) versus , (C), (G) e (L) imersão tridimensional do atrator da série temporal da (D) radiação fotossinteticamente ativa (PARi), (H) evapotranspiração (Et) e (M) troca líquida de $\mathrm{CO} 2$ (CO2flux).

pendentes que participam da dinâmica do sistema. As estimativas da $D c$ e $m$ das séries de temperatura do ar, umidade relativa do ar, radiação solar global, fluxo de $\mathrm{CO}_{2}$ e concentração de $\mathrm{CO}_{2}$ evidenciam a existência de acoplamento desses subsistemas não lineares de baixa dimensão governados por atratores estranhos, dado o seu número e a variedade de subsistemas em interação (Tabela 1). Os altos valores de $D c$ e $m$ das séries da radiação fotossinteticamente ativa e evapotranspiração evidenciam a existência de um acoplamento entre essas duas variáveis, visto a dependência com a dinâmica da resistência estomática, ou seja, com a abertura e fecha- mento dos estômatos da vegetação.

Esperava-se valores próximos de $D c$ e $m$ das séries da radiação solar global e da radiação fotossinteticamente ativa (Tabela 1), visto que essas variáveis são fortemente correlacionadas devido à única fonte delas. No entanto, observa-se menores valores de $D c$ e $m$ da série da radiação solar global. Possivelmente, os padrões de cobertura do céu tenham mais influência sobre uma fração da radiação solar (PARi) do que o todo do espectro que atinge o solo (RG), mas essa hipótese ainda exige mais investigações.

Os valores obtidos para $D c$ são consistentes com diversos 


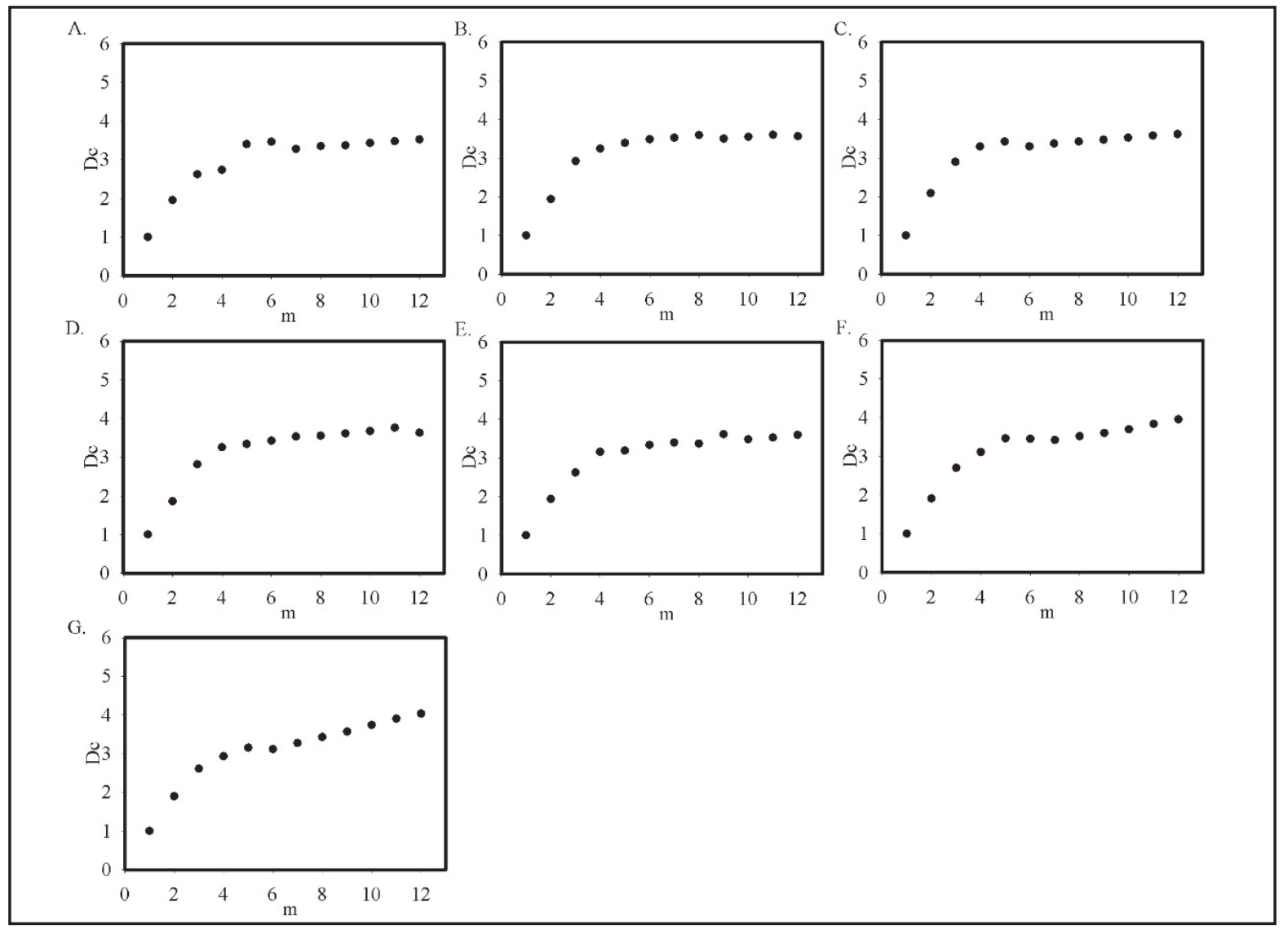

Figura 8: versus das concentrações de $\mathrm{CO}_{2}$ para as alturas: (A) $35 \mathrm{~m}$, (B) $33 \mathrm{~m}$, (C) $32 \mathrm{~m}$, (D) $29 \mathrm{~m}$, (E) $20 \mathrm{~m}$, (F) $12 \mathrm{~m}$ e (G) $3 \mathrm{~m}$.

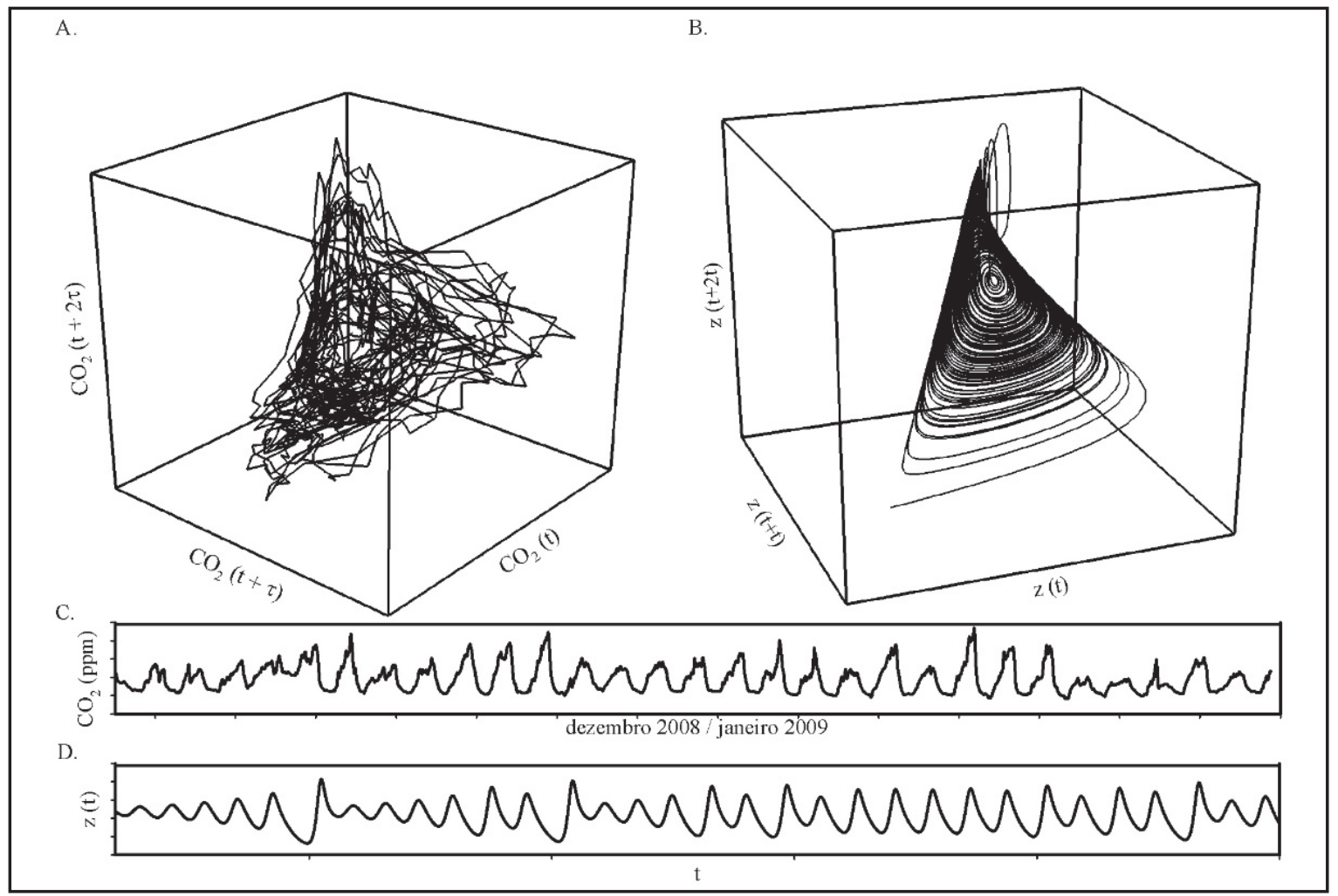

Figura 9 - (A) Imersão tridimensional do atrator do CO2 (concentração a 35 m de altura), e (B) reconstrução do atrator de Lorenz a partir da variável z, com suas respectivas séries temporais (C) e (D) 
Tabela 2 - Valores da dimensão fractal (Dc) e da dimensão de imersão $(\mathrm{m})$ de séries temporais de temperatura do ar e do fluxo de $\mathrm{CO}_{2}$ (CO2flux) obtidos em trabalhos anteriores

\begin{tabular}{|c|c|c|c|}
\hline Variáveis & Dc & m & Referências \\
\hline Temperatura & 3,05 & 6 & Este trabalho \\
\hline Temperatura & 3,50 & 8 & $\begin{array}{c}\text { CAMPANHARO } \\
\text { et al. (2008) }\end{array}$ \\
\hline Temperatura & 4,50 & - & $\begin{array}{l}\text { JARAMILLO e } \\
\text { PUENTE (1993) }\end{array}$ \\
\hline Temperatura & 3,26 & - & XIN et al. (2001) \\
\hline Temperatura & 3,50 & - & $\begin{array}{c}\text { ZENG e PIELKE } \\
\text { (1993) }\end{array}$ \\
\hline $\mathrm{CO}_{2}$ flux & 5,94 & 8 & Este trabalho \\
\hline $\mathrm{CO}_{2 f l u x}$ & 2,70 & 7 & Gallon et al. (2006) \\
\hline
\end{tabular}

trabalhos (Tabela 2) que encontraram atratores de baixa dimensão para séries de temperatura do ar. No entanto, os resultados obtidos de $D c$ de séries de fluxo de $\mathrm{CO}_{2}$ diferem um pouco, provavelmente pela diferença entre os ecossistemas estudados em cada um dos trabalhos. Gallon et al. (2006) analisaram dados provenientes de floresta de transição Amazônia-Cerrado, enquanto que nesse trabalho foi analisado dados provenientes de floresta sazonalmente inundada no Pantanal. Enquanto que os valores do parâmetro m, que significa o número de variáveis independentes necessárias para modelar a dinâmica do fluxo de $\mathrm{CO}_{2}$, os valores foram próximos.

Atratores são regiões restritas do espaço de fase para onde as trajetórias dos estados do sistema convergem. Em sistemas com dinâmica aleatória ou estocástica, o atrator do sistema preenche o espaço de fase inteiramente, isto é, não há organização temporal, a dinâmica do sistema é totalmente imprevisível, enquanto que, em um sistema complexo sua dinâmica fica restrita a uma região do espaço de fase (PEITGEN et al., 2004).

Como os valores de $m$ maiores que três inviabilizam a visualização dos atratores reconstruídos no hiperespaço, apresentamos para efeito de ilustração, uma imersão tridimensional dos atratores (Figuras 6C, 6G, 6L, 7C, $7 \mathrm{G}$ e 7L) associados á cada uma das séries temporais (Figuras 6D, 6H, 6M, 7D, 7H e 7M) com seu $\tau$ adequado (Tabela 1).

A $D c$ da concentração de $\mathrm{CO}_{2}$ foi de $3,50 \pm 0,09$ para quase todo o perfil da torre, exceto para as medidas feitas a 3 e 12 metros (Figura $8 \mathrm{G}$ e $8 \mathrm{~F}$, respectivamente) que apresentaram $D c$ maior que 4 e dimensão de imersão maior que 12. Estes valores elevados são possivelmente devido à proximidade com as fontes da respiração microbiológica do solo (SANTOS e COSTA, 2003; GOMES e VARRIALE, 2004), visto que foi obtido valores menores para as outras medidas dentro e sobre o dossel vegetativo, valores mais vinculados à fisiologia vegetal e atividade fotossintética da floresta.
O atrator da concentração de $\mathrm{CO}_{2}$ (Figura 9A) e o atrator de Lorenz reconstruído a partir da variável Z (Figura 9B) e suas séries temporais (Figura 9C e 9D) apresentam semelhanças topológicas entre o real e o teórico, seja no comportamento das órbitas, nas transições entre picos e nos períodos de frequências estáveis. Disso supõe-se que a equação que rege a estrutura geométrica da dinâmica desses dois sistemas também seja semelhante. Dessa forma, por meio do método conhecido como Lorenz Map é possível predizer um $\mathrm{Zn+1}$ a partir de $\mathrm{Zn}$, ou seja, Zn+1=f(Zn) (STROGATZ, 2000), de modo que ajustes na equação Z de Lorenz (1963) do fluxo determinístico pode vir a modelar a concentração de $\mathrm{CO}_{2}$ na copa da floresta de cambarazal no Pantanal, no período chuvoso antes da inundação.

\section{Conclusão}

As estimativas da dimensão fractal $D c$ e dimensão de imersão $m$ das variáveis evidenciam a existência de acoplamento de subsistemas não lineares de baixa dimensão governados por atratores estranhos, dado o seu número e a variedade de subsistemas em interação.

A maioria das séries temporais de $\mathrm{CO}_{2}$ sofreram uma saturação da $D c$ versus $m$, indicando que o sistema possui um atrator que é acoplado tanto a subsistemas não lineares de baixa dimensão (solo e plantas), quanto a um sistema maior e mais complexo (atmosfera). Estes resultados reforçam a incorporação de aspectos da fisiologia vegetal e microbiologia de solos na modelagem de ecossistemas.

\section{Agradecimentos}

A pesquisa foi apoiada pela Universidade Federal de Mato Grosso (UFMT), Programa de Pós Graduação em Física Ambiental (PPGFA/IF/UFMT), e Coordenação de Aperfeiçoamento de Pessoal do Ensino Superior (CAPES - Bolsas, Processo no 9750/13-4 e nº 9768/13-0), e financiada pelo Conselho Nacional de Desenvolvimento Científico e Tecnológico (CNPq - MCT/CNPq/ CT Infra-CT Energia 2006; processo no 620082/20062), e Fundação de Amparo à Pesquisa do Estado de Mato Grosso (FAPEMAT - PRONEX 2009, processo n ${ }^{0}$ 823971/2009; FAPEMAT - UNIVERSAL 2012, processo no 331763/2012).

\section{Referências}

ARAÚJO, A. C., DOLMAN, A. J., WATERLOO, M. J., GASH, J. H. C., KRUIJT, B., ZANCHI, F. B., LANGE, J. M. E., STOEVELAAR, R., MANZI, A. O., NOBRE, A. D., LOOTENS, R. N., BACKER, J. (2010). The spatial variability of $\mathrm{CO} 2$ storage and the interpretation of eddy 
covariance fluxes in central Amazonia. Agricultural and Forest Meteorology, 150,226-237.

ARIEIRA, J., NUNES DA CUNHA, C. (2006). Fitossiologia de uma floresta inundável monodominante de Vochysia divergens Pohl (Vochysiaceae), no Pantanal Norte, MT, Brasil. Acta Botanica Brasilica, 20,(3),569-580.

BAKER, G. L., GOLLUB, J. P. (1996). Chaotic dynamics: an introduction. 1a edn. Cambridge University Press.

BIUDES, M. S., CAMPELO JÚNIOR, J. H., NOGUEIRA, J. S., SANCHES, L. (2009). Estimativa do balanço de energia em cambarazal e pastagem no norte do pantanal pelo método da razão de Bowen. Revista Brasileira de Meteorologia, 24(2),135-143.

BIUDES, M. S., NOGUEIRA, J. S., DALMAGRO, H. J., MACHADO, N. G., DANELICHEN, V. H. M., SOUZA, M. C. (2012). Mudança no microclima provocada pela conversão de uma floresta de cambará em pastagem no norte do Pantanal. Revista de Ciências Agro-Ambientais, 10,61-68.

BIUDES, M.S., VOURLITIS, G. L., MACHADO, N. G., ARRUDA, P.H.Z., NEVES, G.A.R., LOBO, F.A., NEALE, C. M. U., NOGUEIRA, J.S. (2015). Patterns of energy Exchange for tropical ecosystems across a climate gradiente in Mato Grosso, Brazil. Agricultural and Forest Meteorology, 202,112-124.

CAMPANHARO, A. S. L. O., RAMOS, F. M., MACAU, E. E. N., ROSA, R. R., BOLZAN, M. J. A., SÁ, L. D. A. (2008). Searching chaos and coherent structures in the atmospheric turbulence above the Amazon forest. Philosophical Transactions of The Royal Society A, 366,579-589.

COLLISCHONN, W., TUCCI, C. E. M., CLARKE, R. T. (2001). Further evidence of changes in the hydrological regime of the river Paraguay: part of a wider phenomenon of climate change? Journal of Hydrology, 245,218-238.

GALLEGO, M. C., GARCIA, J.A., CANCILLO, M. L. (2001). Characterization of atmospheric turbulence by dynamical systems techniques. Boundary-Layer Meteorology, 100,375-392.

GALLON, M. M. P., SANCHES, L., DE PAULO, S. R. (2006). Fluxo e perfil de dióxido de carbono no dossel uma floresta tropical de transição amazônica. Revista Brasileira de Meteorologia, 21,(3b),291-300.

GOMES A. G., VARRIA LE M. C. (2004). Modelagem de Ecossistemas: Uma Introdução. 1a edn. Editora da Universidade Federal de Santa Maria.
GRACE, J., MALHI, Y., LLOYD, J., MCINTYRE, J., MiRANDA, A. C., MEIR, P., MIRANDA, H.S. (1996). The Use of Eddy Covariance to Infer the Net Carbon Dioxide Uptake of Brazilian Rain Forest. Global Change Biology, 2,209-217.

GRASSBERGER, P., PROCACCIA, I. (1983). Characterization of strange attractors. Physical Review Letters, 50(5),346-349.

HU, S., ZHAO, C., LI, J., WANG, F., CHEN, Y. (2013). Discussion and reassessment of the method used for accepting or rejecting data observed by a Bowen ratio system. Hydrological Processes, 28(15),4506-4510.

JARA Millo, G. P., PUENTE, C. E. (1993). Strange attractors in atmospheric boundary-layer turbulence. Boundary-Layer Meteorology, 64,175-197.

JUNK W.J. (2002). Long-term environmental trends and the future of tropical wetlands. Environmental Conservation, 29(4),414-435.

JUNK, W. J., NUNES DA CUNHA, C. (2005). Pantanal: a large South American wetland at a crossroads. Ecological Engineering, 24,391-401.

JUNK, W. J., NUNES DA CUNHA, C., WANTZEN, K. M., PETERMANN, P., STRÜSSMANN, C., MARQUES, M. I., ADIS, J. (2006). Biodiversity and its conservation in the Pantanal of Mato Grosso, Brazil. Aquatic Sciences, 68,278-309.

LORENZ, E. N. Deterministic nonperiodic flow. Journal of Atmospheric Sciences, 20,130-141.

MELlO, G. J., PAUlO, I. J. C., PAUlO, S. R., GOMES, R.S.R., MACHADO, N. G., NOGUEIRA, J.S., BIUDES, M. S. (2013). Dimensão fractal de séries temporais acima do dossel de floresta no Pantanal matogrossense. Revista Brasileira de Climatologia, 12,61-83.

MOORE, C. J., FISCH, G. (1986). Estimating heat storage in Amazonian tropical forest. Agricultural and Forest Meteorology, 38(1-3),147-168.

NEUE, H. U., GAUNT, J. L., WANG, Z.P., BECHERHEIDMANN, P., QUIJANO, C. (1997). Carbon in tropical wetlands. Geoderma, 79,163-185.

NUNES D A CUNHA, C., JUNK, W. J. (2004). Yearto-year changes in water level drive the of Vochysia divergens in Pantanal glassland. Applied Vegetation Science, 7,103-110.

ORTEGA-FARIAS, S. O., CUENCA, R. H., EK, M. (1996). Daytime variation of sensible heat flux estimated 
by the bulk aerodynamic method over a grass canopy. Agricultural and Forest Meteorology, 81,131-143.

PEITGEN, H., JORGENS, H., SAUPE, D. (2004). Chaos and fractals: new frontiers of science. 1a edn. Springer-Verlag.

PEREZ, P. J., CASTELLVI, F., IBANEZ, M., ROSELL, J. I. (1999). Assessment of reliability of Bowen ratio method for partitioning fluxes. Agricultural and Forest Meteorology, 97,141-150.

SANTOS, S. N. M., COSTA, M. H. (2003). Simulações de fluxo de carbono em um ecossistema de floresta tropical. Revista Brasileira de Meteorologia, 18(1),87-96.

STROGATZ, S. H. (2000). Nonlinear dynamics and Chaos. 1a edn. Westview Press.

TAKENS, F. (1981). Detecting Strange Attractors in Turbulence. Lecture Notes in Mathematics, 898,366-381.

UNLAND,H.E.,HOUSER, P.R.,SHUTTLEWORTH, W. J., YANG, Z. L. (1996). Surface flux measurement and modelling at a semi-arid Sonoran Desert site. Agricultural and Forest Meteorology, 82, 119-153.

VOURLITIS, G. L., LOBO, F. A., BIUDES, M. S., ORTÍZ, C. E. R., NOGUEIRA, J. S. (2011). Spatial variations in soil chemistry and organic matter content across a Vochysia divergens invasion front in the Brazilian Pantanal. Soil Science Society of America Journal, 75,15541561.

WOLF, A., SALIENDRA, N., AKSHALOV, K., JOHNSON, D.A., LACA, E. (2008). Effects of different eddy covariance correction schemes on energy balance closure and comparisons with the modified Bowen ratio system. Agricultural and Forest Meteorology, 48,942-952.

XIN, L., FEI, H., GANG, L. (2001). Characteristics of chaotic attractors in atmospheric boundary layer turbulence. Boundary-Layer Meteorology, 99,335-345.

ZENG, X., PIELKE, R. A. (1993). What does a lowdimensional weather attractor mean? Physics Letters A, 175,299-304. 